\title{
Uso da simulação computacional para ensino e aprendizagem nos cursos de saúde
}

\author{
Use of computer simulation for teaching and learning in health courses \\ Uso de simulación por computadora para la enseñanza y el aprendizaje en cursos de salud
}

Recebido: 18/11/2021 | Revisado: 23/11/2021 | Aceito: 25/11/2021 | Publicado: 26/11/2021

Gutemberg Ferreira de Almeida

ORCID: https://orcid.org/0000-0001-5730-6826 Faculdade Santa Maria, Brasil E-mail: gutemberg.ferreiraalmeida@gmail.com Lorena de Souza Sobral ORCID: https://orcid.org/0000-0001-9845-2600 Faculdade Santa Maria, Brasil

E-mail: lorenassobral1@gmail.com

José de Arimateia Oliveira

ORCID: https://orcid.org/0000-0001-6501-3927 Faculdade Santa Maria, Brasil E-mail: arimateioliveirasantiago@ hotmail.com Beatriz Torres Baltazar ORCID: https://orcid.org/0000-0001-9478-5969 Faculdade Santa Maria, Brasil

E-mail: beatriztbaltazar@hotmail.com

Alexandrina Silveira Neta

ORCID: https://orcid.org/0000-0002-7748-2074 Faculdade Santa Maria, Brasil

E-mail: alexandrinaslvn@gmail.com Jáiron José Tavares

ORCID: https://orcid.org/0000-0002-2429-0458 Faculdade Santa Maria, Brasil

E-mail: jairontavares5@hotmail.com

Bárbara Fernandes de Novais

ORCID: https://orcid.org/0000-0002-8337-6876 Faculdade Santa Maria, Brasil

E-mail: fernandes.barbara@hotmail.com

Renan Marques Gadelha Rodrigues

ORCID: https://orcid.org/0000-0001-8674-3910 Faculdade Santa Maria, Brasil

E-mail: renangadelha@yahoo.com.br

João Marcos Marinho Cavalcanti ORCID: https://orcid.org/0000-0003-1117-8026

Universidade Federal de Pernambuco, Brasil E-mail: joaoufpe44@gmail.com

Lívia Andrade Duarte

ORCID: https://orcid.org/0000-0003-0312-3084

Centro Universitário Atenas, Brasil

E-mail: livia0593@yahoo.com.br

Gabriela Fonseca Marçal

ORCID: https://orcid.org/0000-0002-9476-6650

Centro Universitário Atenas, Brasil

E-mail: gabrielafonsecamarcal@gmail.com

Raniery Soares Sobreira Machado

ORCID: https://orcid.org/0000-0003-1478-948X

Hospital da Restauração Gov. Paulo Guerra, Brasil

E-mail: raniery.machado@hotmail.com

\section{Resumo}

A humanidade passa por uma movimentação tecnológica nunca vista até o momento, em que a informação e a comunicação alcançam um plano essencial na vida das pessoas. Dentre as inovações tecnológicas, destaca-se as simulações computacionais, no qual pode ser conceituada como a construção e manipulação operacional de um modelo, ou seja, uma representação física ou simbólica de aspectos de um processo físico e ou social. A simulação computacional é aplicada nos cursos de saúde no ensino de técnicas e procedimentos clínicos, incluindo diversos objetos: jogos, modelos anatômicos, manequins, bonecos, estudos de casos e apresentações de multimídia. As simulações facilitam a aquisição de habilidades para os cuidados aos pacientes, propiciando a imersão dos estudantes 
em ambientes interativos seguros para desenvolver o processo de saúde. Neste sentido, o presente artigo tem como objetivo realizar um estudo exploratório e de caráter bibliográfico sobre o uso da simulação computacional para ensino e aprendizagem nos cursos de saúde, evidenciando os principais benefícios dessa tecnologia em faculdades no século XXI.

Palavras-chave: Laboratório virtual; Simulação; Saúde.

\begin{abstract}
Humanity is going through a technological movement never seen before, in which information and communication reach an essential level in people's lives. Among the technological innovations, computer simulations stand out, which can be conceptualized as the construction and operational manipulation of a model, that is, a physical or symbolic representation of aspects of a physical or social process. Computer simulation is applied in health courses to teach clinical techniques and procedures, including various objects: games, anatomical models, mannequins, dolls, case studies, and multimedia presentations. Simulations facilitate the acquisition of skills for patient care, providing the immersion of students in safe interactive environments to develop the health process. In this sense, this article aims to conduct an exploratory and bibliographic study on the use of computer simulation for teaching and learning in health courses, highlighting the main benefits of this technology in colleges in the 21 st century.
\end{abstract}

Keywords: Virtual laboratory; Simulation; Health.

\title{
Resumen
}

La humanidad atraviesa un movimiento tecnológico nunca antes visto, en el que la información y la comunicación alcanzan un nivel esencial en la vida de las personas. Entre las innovaciones tecnológicas, se destaca como simulaciones computacionales, que pueden conceptualizarse como la construcción y manipulación operativa de un modelo, es decir, una representación física o simbólica de aspectos de un proceso físico y / o social. Se aplica una simulación por computadora en los cursos de salud para enseñar técnicas y procedimientos, incluyendo varios objetos: juegos, modelos anatómicos, maniquíes, títeres, estudios de casos y presentaciones multimedia. Como simulaciones, facilitan la adquisición de habilidades para la atención al paciente, proporcionando a los estudiantes una inmersión en entornos de seguros interactivos para desarrollar el proceso de salud. En este sentido, este artículo tiene como objetivo realizar un estudio exploratorio y bibliográfico sobre el uso de la simulación informática para la enseñanza y el aprendizaje en los cursos de salud, destacando los principales beneficios de esta tecnología en las universidades del siglo XXI.

Palabras clave: Laboratorio virtual; Simulación; Salud.

\section{Introdução}

A tecnologia não é algo novo para a humanidade. Desde os primórdios da sua existência, o homem cria e produz tecnologias para satisfazerem suas necessidades. A descoberta do fogo, a invenção da escrita e a utilização da roda, são fatos que marcaram o início da civilização humana, com a evolução da humanidade, outros elementos que poderiam ser considerados tecnológicos foram surgindo e um dos principais foi à invenção da imprensa pelo alemão Gutemberg, o qual marcou uma revolução tecnológica no campo da literatura e imprensa, tendo a Bíblia como seu primeiro livro imprenso (Lopes, 2017).

O desenvolvimento da humanidade é acompanhada diretamente pelo avanço das tecnologias, de natureza e objetivos variados, podendo facilitar, otimizar ou adaptar uma determinada tarefa ou realidade vivida em um determinado tempo da história, como por exemplo, temos os avanços consideráveis nos vários segmentos na área da saúde, como a qualidade de vida e bem estar, ou seja, cada vez mais é buscado a compreensão das necessidades das pessoas, juntamente com métodos propostos para suas resoluções, sem deixar o desenvolvimento dos demais setores, comercial, industrial e energético (Lorenzetti et al., 2012).

O surgimento da internet, ocorrido em tempos de guerra, como uma forma de vantagem estratégica para a comunicação, hoje nos permite usufruir de facilidades em escala nunca vistas antes, em velocidade praticamente imediata. Pois, rompe o limite geográfico e nos confere a capacidade de nos comunicarmos com pessoas em outros estados, países e continentes, fato que propicia cada vez maior contribuição e compartilhamento de material técnico-científico, devido a união de pesquisas feitas por autores com experiências, técnicas e visões particulares (Rocha \& Souza Filho, 2016). 
Quando se fala em tecnologia na educação, logo se pensa em computadores, internet, mas isso não é tudo, tecnologia é, efetivamente, mais do que isso. Ela se faz presente, por exemplo, em todos os lápis que se usa, no quadro de giz, nos livros, nas cadeiras em que se senta. Pode-se imaginar uma escola, hoje sem livros, sem material impresso?

Para Sabbadini e Gonçalves (2004), nas últimas décadas a aplicação da simulação computacional tem demonstrado ser esta uma técnica poderosa, especialmente no ensino e aprendizagem. De acordo com Pegden e Rosenshine (1990), "a simulação é um processo de projetar um modelo computacional de um sistema real e conduzir experimentos com este modelo com o propósito de entender seu comportamento e/ou avaliar estratégias para sua operação”. Law e Kelton (2000) complementa ao afirmar que a simulação é uma técnica que usa computadores para imitar as operações de diversos tipos de processos e facilidades do mundo real. Ou seja, é a imitação da operação de um processo ou sistema do mundo real.

Peres, Suzuki e Azevedo-Marques (2015) afirmam que a partir da década de 1990, verifica-se um aumento significativo no número de estudos sobre a educação intercedida por tecnologias na área da saúde e suas diversas ferramentas e recursos.

De acordo com Lee, Choi e Jeon (2021), a simulação computacional melhora os resultados do aprendizado dos estudantes, como aquisição de conhecimento e desempenho. Em particular, a educação baseada em simulação utilizando pacientes padronizados tem sido amplamente utilizada como uma alternativa para a colocação clínica, proporcionando exercícios práticos para melhorar as habilidades técnicas dos estudantes.

A simulação pode ser definida como a construção e manipulação operacional de um modelo, ou seja, uma representação física ou simbólica de aspectos de um processo físico e ou social (Júnior, Souza \& Nascimento, 2012). Através de simulações computacionais, é possível criar as condições indispensáveis para que a aprendizagem significativa aconteça, em que as atividades de modelagem e simulação estão entre as maneiras mais criativas, benéficas e que geram interação de aplicações na Educação (Souza \& Dandolini, 2009).

De acordo com Teixeira e Felix (2011), a simulação computacional é aplicada nos cursos de saúde no ensino de técnicas e procedimentos clínicos, incluindo diversos objetos: jogos, modelos anatômicos, manequins, bonecos, estudos de casos e apresentações de multimídia. As simulações facilitam a aquisição de habilidades para os cuidados aos pacientes, propiciando a imersão dos estudantes em ambientes interativos seguros para desenvolver o processo de saúde. Para os autores, as simulações oferecem experiências cognitivas, psicomotoras e afetivas, corroborando para a transferência de conhecimento da sala de aula para os ambientes clínicos.

Sendo assim, o presente artigo tem como objetivo realizar uma revisão de literatura sobre o uso da simulação computacional para ensino e aprendizagem nos cursos de saúde, evidenciando os principais benefícios dessa tecnologia em faculdades no século XXI. Este trabalho justifica-se pela necessidade de fomentar essa temática pouco abordada na literatura, apesar de sua grande importância nas ciências da saúde e educacionais.

\section{Metodologia}

Trata-se de um estudo descritivo do tipo revisão bibliográfica, no qual se proporcionou uma síntese de conhecimento e a incorporação dos resultados de estudos significativos que ocorreram através de levantamento bibliográfico baseado na experiência vivenciada de outros autores.

Para Gil (2010) o estudo bibliográfico é tido desde a concepção do projeto e organização de ideias as compreende etapas (formulação do problema, a escolha do tema a ser investigada, a elaboração do plano de trabalho, a formulação dos objetivos, identificação, localização e obtenção de fontes que sejam capazes de fornecer os dados adequados à pesquisa desejada, leitura do material obtido, análise e interpretação lógica dos dados e redação final do texto). Desta forma, para o 
autor a pesquisa bibliográfica não está detida apenas no levantamento de dados, neste sentido o estudo bibliográfico é bem mais amplo.

Em sentido mais amplo, este tipo de pesquisa se resume em um levantamento de dados para solucionar uma problematização de um projeto referenciando autores e dados já previamente estudados e publicados pela comunidade científica, sendo ainda uma ancoragem de bagagem para embasamento teórico, habilitando o indivíduo a produção de trabalhos (Gil, 2010).

Para a realização desse estudo, foram utilizados os descritores validados através dos Descritores em Ciências da Saúde (DECS), o vocabulário DECS contém a terminologia padrão em ciências da saúde, em português, espanhol e inglês. "O DECS é um vocabulário dinâmico e em constate ampliação. Sua manutenção e atualização é efetuada em estrutura de base de dados" (Castro, 2001, p.02).

Foi utilizado para a indexação e navegações nas fontes de informações da Biblioteca Virtual em Saúde, foram criados pelo Centro Latino Americano e do Caribe de Informações em Ciência e Saúde BIREME/OPAS/OMS, tudo com intuito de facilitar a indexação de publicações científicas. Desta forma, para análise desta pesquisa, foi utilizada a base do DECS de acordo com a Biblioteca Virtual em Saúde: Simulação Computacional, Ensino, Aprendizagem e Saúde.

A pesquisa foi realizada na base de dados da Biblioteca Virtual de Saúde (BVS) por representar esta plataforma uma dissociação de conhecimentos científicos que surge das preocupações dos órgãos públicos nacionais e internacionais por oferecer através da internet uma informação científica de qualidade garantida. Nesta plataforma estão indexadas também outras bibliotecas virtuais, tais como SciELO (Scientific Electronic Library Online), BIRENE (Biblioteca Regional de Medicina), BDENF (Base de dados de enfermagem), LILACS (Literatura Latino-Americana e do Caribe em Ciências da Saúde), MEDLINE (Literatura Internacional em Ciências da Saúde), Coleciona SUS (Coleção Nacional das Fontes de Informação do SUS), MS (Acervo da Biblioteca do Ministério da Saúde), SAÚDE LEGIS (Legislação Federal da Saúde), OPAS (Acervo da Biblioteca da Organização Pan-Am. Saúde), WHOLIS (Sistema de Informação da Biblioteca da OMS).

Quanto ao critério de delimitação do tema, estes compreenderam os últimos 10, haja vista, que se pretendeu fazer um levantamento do maior número de produções sobre a temática. As vantagens em trabalhar com esse tipo de pesquisa, estão justamente na possibilidade de maior aquisição de dados recentes, deixando assim a pesquisa mais robusta, onde pode-se confrontar diversas opiniões de diversos autores.

Com a leitura dos resumos e artigos metodológicos e mediante pertinência dos conteúdos foram observados os critérios de inclusão, ou seja, os estudos e dados disponíveis na íntegra, em acesso livre, publicações oriundas da língua portuguesa e inglesa. Como critério de exclusão se optou: revistas Maguezine, artigo noticiosos, textos em resenhas, artigos não indexados, opiniões e editoriais ou manuais. Os procedimentos metodológicos estão presentes na Figura 1.

Figura 1: Procedimentos metodológicos. 


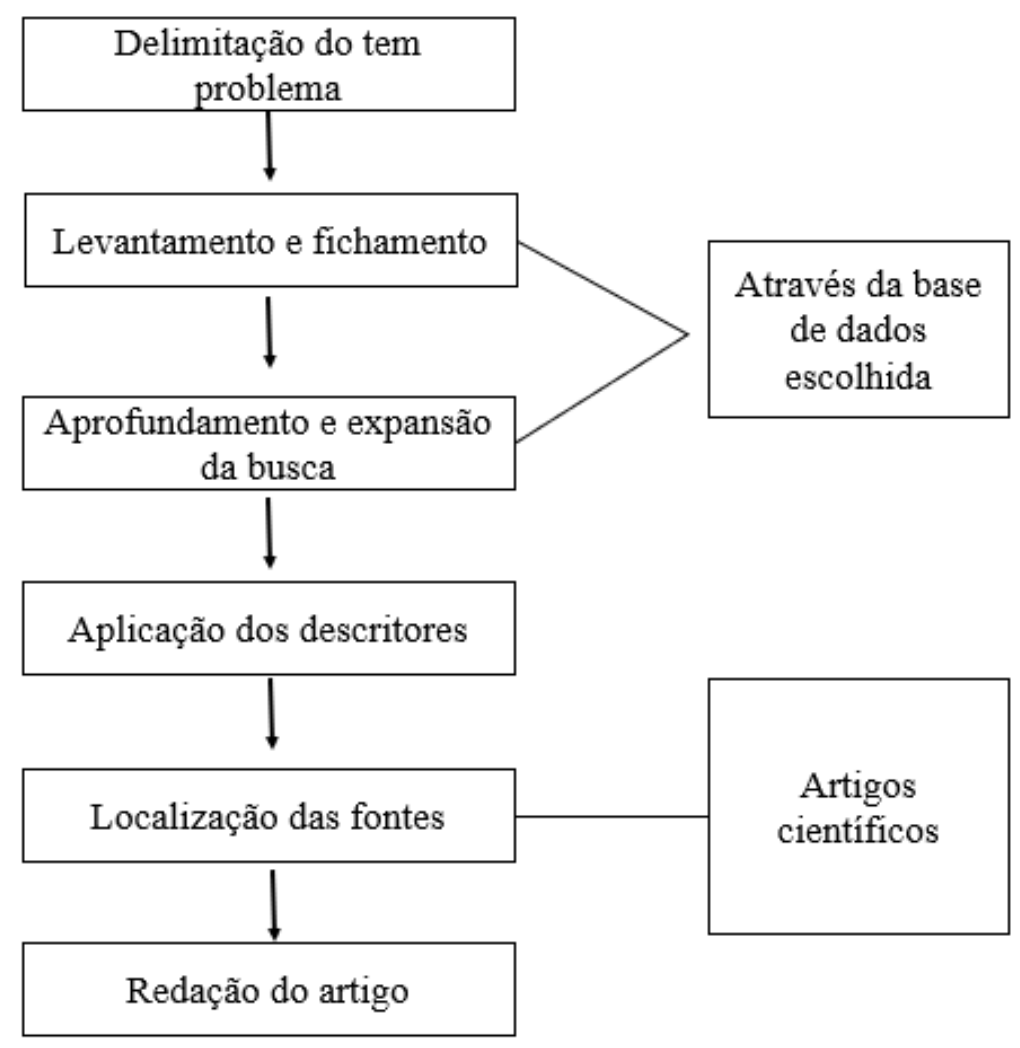

Fonte: Autores (2021).

Operacionalmente, foi adotada para a análise de dados a Análise Temática de Conteúdo, informações que segundo Minayo (2010), desdobra-se nas etapas pré-análise, exploração do material ou codificação e tratamento dos resultados obtidos/ interpretação.

A etapa da pré-análise compreende a formulação e reformulação de hipóteses ou pressupostos. Durante a etapa da exploração do material, o investigador busca encontrar categorias que são expressões ou palavras significativas em função das quais o conteúdo de uma fala será organizado. A categorização consiste num processo de redução do texto às palavras e expressões significativas. A partir daí, o analista propõe inferências e realiza interpretações, inter-relacionando-as com o quadro teórico desenhado inicialmente ou abre outras pistas em torno de novas dimensões teóricas e interpretativas, sugerida pela leitura do material (Minayo, 2010).

\section{Resultados e Discussões}

A simulação computacional emprega formalizações em computadores, tais como expressões matemáticas ou especificações formalizadas, objetivando imitar um processo ou operação do mundo real (Santos \& MOURA, 2019; Walczak \& Moura, 2019). Segundo os autores, a simulação computacional usa técnicas matemáticas, no qual permitem imitar o funcionamento de praticamente qualquer tipo de operação ou processo do mundo real. Sendo assim, é possível analisar o comportamento de sistemas reais por meio de modelos matemáticos virtuais, para definir dados reais e conduzir experimentos com o propósito de entender o comportamento de tal fenômeno.

Medeiros, Moser e Santos (2015) afirmam que a simulação pode ter aplicações de diversos tipos: dispositivo entender um problema; uma forma de comunicação para apresentar a operação de um sistema; um instrumento crítico para produzir elementos e aferir medidas de desempenho; uma ferramenta de projeto para avaliar problemas e propor soluções; um sistema 
de planejamento de operações para trabalhos, tarefas e recursos; um mecanismo de controle; um instrumento de treinamento; uma parte do sistema para fornecer informações, projeções de situações e apoio à decisão.

A construção desenvolvimento de um modelo pode ser complicado e exigir estudo e análise, como também pode ser simples, a depender do seu objetivo. A simulação tem a finalidade de descrever algo do mundo real através de números. Ao desenvolve-lo, é necessário considerar: Quais fenômenos estão envolvidos? Quais são os dados necessários? Quais são os dados esperados? Quanto de precisão os resultados irão possuir? Quem vai utilizá-lo? Quais riscos ele pode trazer? (BANKS, 2000).

Christie et al. (2017) destaca que os resultados de uma simulação computacional indicam o desempenho dos processos ou operações, pela possibilidade de utilização de uma combinação de modelos, bem como pela visualização dos dados de forma tridimensional, permitindo, assim, uma melhor identificação do alcance das atividades. Vale destacar que o uso da simulação evita custos com a investigação científica experimental, visto que a modelagem computacional permite uma visão clara das estimativas nos pontos desejáveis de um processo.

A simulação na área da saúde tornou-se uma parte essencial da educação de estudantes e profissionais de saúde na prática. Muitas instituições fizeram recomendações sobre o uso de simulação na formação em saúde (Aebersold \& Tschannen, 2013). A utilização das simulações computacionais é uma estratégia de cunho didático que pode ser utilizada no ensino superior e se organiza de forma que os discentes aprendam em uma situação similar a real, aproximando os conceitos trabalhados em sala de aula da realidade, promovendo o aprendizado do aluno, possuindo vantagens como: facilidade da aquisição de conceitos, a substituição de laboratórios experimentais, o aprendizado sem riscos e o fato de atrair a atenção do aluno (Barros, 2015).

Em um estudo realizado por Stirling, Smith e Hogg (2012), verificaram que a simulação computacional tem demonstrado benefícios na enfermagem no preparo de enfermeiras recém-formadas para o ambiente de prática. O estudo piloto foi realizado no Hospital Ninewells na Escócia, em conjunto com a Universidade de Dundee, um programa de simulação de enfermaria foi desenvolvido com foco no desenvolvimento das capacidades de enfermeiras recém-qualificadas. Um grupo foi conduzido após as simulações serem concluídas e os seguintes temas foram identificados: aumento na confiança, desenvolvimento de habilidades de gerenciamento de estresse, gerenciamento aprimorado do paciente agudamente indisposto.

Em um estudo com objetivo de verificar os benefícios dessa tecnologia no ensino e aprendizagem em Ciências da Saúde, Harder (2010) verificou que o uso de simulação como uma ferramenta educacional está se tornando cada vez mais prevalente na prática de saúde e foi reconhecida como um método de ensino que usa exercícios de aprendizagem que imitam de perto as situações da vida real. O desenvolvimento de cenários de simulação clínica com base em evidências e diretrizes para profissionais de saúde é um passo importante no redesenho da educação.

Esses cenários são criados para que os alunos aprendam em um ambiente seguro. A experiência clínica simulada requer a imersão dos alunos em um cenário representativo de atendimento ao paciente, um cenário que imita o ambiente real com realismo suficiente para permitir que os alunos suspendam a descrença (Waxman, 2010).

Schlairet e Pollock (2010), em um estudo de caso com finalidade de evidenciar os ganhos da simulação computacional em cursos de saúde, verificaram que, após a atribuição aleatória, os alunos participaram de experiências clínicas simuladas baseadas em laboratório com simuladores de pacientes humanos de alta fidelidade e experiências clínicas tradicionais e completaram pré-testes e pós-testes de conhecimento. A análise identificou ganho de conhecimento significativo associado a experiências clínicas tradicionais e simuladas, ganhos estatisticamente significativamente. A experiência clínica simulada foi considerada tão eficaz quanto a experiência clínica tradicional.

Em um estudo sobre os ganhos com o uso da simulação para a aprendizagem dentro do ensino de enfermagem, Ricketts (2011) verificou que a aprendizagem simulada em um laboratório de habilidades clínicas aumenta a confiança do 
aluno e os prepara para um ambiente clínico real. Para o autor, uma abordagem padronizada para a aprendizagem simulada no ensino de enfermagem e o desenvolvimento de mais cenários clínicos holísticos que estão relacionados à teoria, ofereceria resultados de aprendizagem mensuráveis para atender aos requisitos profissionais e regulamentares. Logo, pode garantir oportunidades de aprendizagem de qualidade, que sejam flexíveis e responsivas às suas necessidades e às demandas dos Serviços de Saúde.

Barros (2015) utilizou a simulação computacional no ensino e aprendizagem de Farmacologia, no qual verificou uma redução do número de experimentos com animais, que necessita de uma aprovação do Comitê de Ética em pesquisa da IES, bem como facilitou a compreensão pelo aluno dos assuntos teóricos abordados em sala de aula. Antoneli et al. (2018) complementam ao afirmar que a simulação computacional no processo de ensino-aprendizagem na área da saúde compreende o desenvolvimento de técnicas e competências capazes de transformar o ensino, tanto para o docente quanto para os discentes e, consequentemente, impacta de forma direta na formação profissional dos estudantes da área da saúde.

Paiva et al. (2013) estudaram a aplicação da Realidade Virtual e Aumentada aplicada ao estudo da anatomia humana, no qual verificaram que a Realidade Virtual e aumentada pode corroborar e ser utilizada no ensino da anatomia humana, tanto para estudantes de medicina e cursos afins quanto para os pacientes que utilizam os consultórios médicos. Além disso, Aguiar et al. (2021) identificou que a realidade virtual está sendo utilizada como ferramenta para o ensino na área da saúde em diversos âmbitos, como na anatomia, treinamento de habilidades práticas e procedimentos, promovendo a interação do usuário com as aplicações computacionais e proporcionando interação em tempo real, despontando-se com o meio tridimensional realista.

Teixeira e Felix (2011), em um estudo sobre a simulação como estratégia de ensino para estudantes de graduação em enfermagem, no qual verificaram que essa tecnologia é uma estratégia utilizada nas disciplinas dos cursos de saúde para o ensino de técnicas e procedimentos necessários para a realização de cuidados, contribuindo para a prática dos estudantes, na transição de ambientes virtuais e controlados em laboratórios para a assistência aos pacientes, podendo ser adjunta à tecnologia computacional, como apoio ao ensino presencial.

O Quadro 1 apresenta os estudos que foram incluídos no presente estudo, no qual mostra os autores, ano de publicação, título e objetivo dos trabalhos. 
Quadro 1: Estudos incluídos na pesquisa.

\begin{tabular}{|c|c|c|c|}
\hline & Autores & Título & Objetivo \\
\hline 1 & $\begin{array}{c}\text { Aebersold e } \\
\text { Tschannen (2013) }\end{array}$ & $\begin{array}{l}\text { Simulação na prática de } \\
\text { enfermagem: o impacto no } \\
\text { cuidado ao paciente }\end{array}$ & $\begin{array}{l}\text { Apresentar uma visão geral das técnicas e } \\
\text { usos de simulação computacional na saúde }\end{array}$ \\
\hline 2 & Barros (2015) & $\begin{array}{c}\text { O uso de simulações } \\
\text { computacionais em } \\
\text { Farmacologia }\end{array}$ & $\begin{array}{c}\text { Verificar a aplicação da simulação } \\
\text { computacional no ensino e aprendizagem de } \\
\text { Farmacologia }\end{array}$ \\
\hline 3 & $\begin{array}{l}\text { Stirling, Smith e } \\
\text { Hogg (2012) }\end{array}$ & $\begin{array}{l}\text {. Os benefícios de um } \\
\text { exercício de simulação de } \\
\text { enfermaria como uma } \\
\text { experiência de aprendizado }\end{array}$ & $\begin{array}{c}\text { Descrever os resultados de um estudo piloto } \\
\text { realizado para identificar os benefícios } \\
\text { potenciais de um exercício de simulação } \\
\text { computacional no desenvolvimento das } \\
\text { capacidades de enfermeiros recém- } \\
\text { qualificados }\end{array}$ \\
\hline 4 & Harder (2010) & $\begin{array}{l}\text { Uso de simulação no ensino e } \\
\text { aprendizagem em ciências da } \\
\text { saúde }\end{array}$ & $\begin{array}{l}\text { Verificar o uso de simulação como uma } \\
\text { ferramenta educacional na prática de saúde }\end{array}$ \\
\hline 5 & Waxman (2010) & $\begin{array}{c}\text { O desenvolvimento de } \\
\text { cenários de simulação clínica } \\
\text { baseados em evidências: } \\
\text { Diretrizes para educadores de } \\
\text { enfermagem }\end{array}$ & $\begin{array}{c}\text { Discutir o desenvolvimento do Bay Area } \\
\text { Simulation Collaborative de diretrizes para } \\
\text { cenários baseados em evidências eficazes } \\
\text { para uso em hospitais e escolas de } \\
\text { enfermagem. }\end{array}$ \\
\hline 6 & $\begin{array}{l}\text { Schlairet e Pollock } \\
\qquad(2010)\end{array}$ & $\begin{array}{l}\text { Teste de equivalência de } \\
\text { experiências clínicas } \\
\text { tradicionais e simuladas: } \\
\text { Aquisição de conhecimento } \\
\text { de alunos de graduação em } \\
\text { enfermagem }\end{array}$ & $\begin{array}{c}\text { Verificar de forma quantitativa o aprendizado } \\
\text { dos alunos de cursos de saúde a partir da } \\
\text { simulação computacional }\end{array}$ \\
\hline 7 & Ricketts (2011) & $\begin{array}{l}\text { O papel da simulação para a } \\
\text { aprendizagem dentro do } \\
\text { ensino de enfermagem pré- } \\
\text { registro }\end{array}$ & $\begin{array}{l}\text { Verificar os benefícios da simulação } \\
\text { computacional nos cursos de saúde }\end{array}$ \\
\hline 8 & Antoneli et al. (2018) & $\begin{array}{l}\text { O uso de simuladores no } \\
\text { auxílio do ensino- } \\
\text { aprendizagem na enfermagem }\end{array}$ & $\begin{array}{l}\text { Verificar os processos da simulação } \\
\text { computacional no processo de ensino- } \\
\text { aprendizagem na área da saúde }\end{array}$ \\
\hline 9 & Paiva et al. (2013) & $\begin{array}{l}\text { Aplicação para dispositivos } \\
\text { móveis utilizando tecnologias } \\
\text { interativas: a Realidade } \\
\text { Virtual e Aumentada aplicada } \\
\text { ao estudo da anatomia } \\
\text { humana }\end{array}$ & $\begin{array}{c}\text { Estudar a aplicação da Realidade Virtual e } \\
\text { Aumentada aplicada ao estudo da anatomia } \\
\text { humana }\end{array}$ \\
\hline 10 & Aguiar et al. (2021) & $\begin{array}{l}\text { Utilização da realidade virtual } \\
\text { para o ensino em saúde }\end{array}$ & $\begin{array}{c}\text { Verificar a aplicação da realidade virtual } \\
\text { como ferramenta para o ensino na área da } \\
\text { saúde }\end{array}$ \\
\hline 11 & $\begin{array}{l}\text { Teixeira e Felix } \\
\text { (2011) }\end{array}$ & $\begin{array}{l}\text { Simulação como estratégia de } \\
\text { ensino em enfermagem }\end{array}$ & $\begin{array}{l}\text { Estudar a aplicação da realidade virtual como } \\
\text { ferramenta para o ensino na área da saúde }\end{array}$ \\
\hline
\end{tabular}

Fonte: Autores (2021).

\section{Considerações Finais}

O presente artigo buscou realizar uma revisão de literatura sobre o uso da simulação computacional para ensino e aprendizagem nos cursos de saúde, evidenciando os principais benefícios dessa tecnologia em faculdades no século XXI. Verificou-se que o objetivo do trabalho foi cumprido, tendo em vista que foi apresentado 11 estudos que verificaram os benefícios da simulação computacional para a aprendizagem dentro do ensino em saúde.

Averiguou-se através da revisão, o uso de simulação como uma ferramenta educacional está se tornando cada vez mais presente na prática de saúde, sendo reconhecida como um método de ensino que usa exercícios de aprendizagem que imitam de perto as situações da vida real, proporcionando aumento na confiança, desenvolvimento de habilidades de gerenciamento de estresse e gerenciamento aprimorado do paciente agudamente indisposto. 
Research, Society and Development, v. 10, n. 15, e565101523522, 2021

(CC BY 4.0) | ISSN 2525-3409 | DOI: http://dx.doi.org/10.33448/rsd-v10i15.23522

\section{Referências}

Aebersold, M., \& Tschannen, D. (2013). Simulação na prática de enfermagem: o impacto no cuidado ao paciente. The Online Journal of Issues in Nursing, $18(2)$.

Aguiar, B. et al. (2021). Utilização da realidade virtual para o ensino em saúde. REIN-Revista Educação Inclusiva, 5(1).

Antoneli, G. et al. (2018). O uso de simuladores no auxílio do ensino-aprendizagem na enfermagem. Educação \& Linguagem, 21(2), $25-42$.

Banks, J. (2000). Introdução à simulação. In: Anais de conferências de simulação de inverno de 2000 (Cat. No. 00CH37165). IEEE, 9-16.

Banks, J. (1998). Princípios de simulação. Manual de simulação, 12, 3-30.

Barros, W. B. Z. G. (2015). O uso de simulações computacionais em Farmacologia. Revista EDaPECI, 15(1), 74-87.

Christie, J. K. et al. (2017). Estruturas e propriedades de vidros bioativos à base de fosfato de simulação computacional: uma revisão. Journal of Materials Chemistry B, 5(27), 5297-5306.

Gil, A. C. et al. (2002). Como elaborar projetos de pesquisa. Atlas.

Harder, B. N. (2010). Uso de simulação no ensino e aprendizagem em ciências da saúde: uma revisão sistemática. Revista de Educação em Enfermagem, 49(1), 23-28.

Júnior, N. T., De Souza, G., \& Do Nascimento, J. Z. (2012). Análise de processos por meio da simulação computacional: proposta de uma estratégia de ensino na graduação em Administração. Administração: Ensino e Pesquisa, 13(3), 491-521.

Law, A. M., \& Kelton, W. D. (2000). Modelagem e análise de simulação. McGraw-Hill.

Lee, U., Choi, H., \& Jeon, Y. Experiências de Estudantes de Enfermagem com Educação em Comunicação Baseada em Simulação de Computadores. Jornal Internacional de Pesquisa Ambiental e Saúde Pública, 18(6), 3108.

Lopes, F. A. S. (2017). Mídia, arte e tecnologia: uma reflexão contemporânea. Comunicação e Sociedade, 31, $287-298$.

Lorenzetti, J. et al. (2012). Tecnologia, inovação tecnológica e saúde: uma reflexão necessária. Texto \& Contexto-Enfermagem, 21 , $432-439$.

Minayo, M. C. de S., Souza, E. R. de., Paula, D. da R. de. (2010). Revisão sistemática da produção acadêmica brasileira sobre causas externas e violências contra a pessoa idosa. Ciência \& Saúde Coletiva, 15, 2719-2728.

Paiva, L. R. M. et al. (2013). Aplicação para dispositivos móveis utilizando tecnologias interativas: a Realidade Virtual e Aumentada aplicada ao estudo da anatomia humana. In: Encontro Anual de Computação, 10(1), 1-8.

Pegden, C. D., \& Rosenshine, M. (1990). Programação de chegadas às filas. Computadores e Pesquisa Operacional, 17(4), 343-348.

Peres, C. M., Suzuki, K. M. F., \& De Azevedo-Marques, P. M. (2015). Recursos tecnológicos de apoio ao ensino na saúde. Medicina (Ribeirão Preto), 48(3), 224-232.

Ricketts, B. (2011). O papel da simulação para a aprendizagem dentro do ensino de enfermagem pré-registro - uma revisão da literatura. Educação de enfermagem hoje, 31(7), 650-654.

Rocha, G. C. da., \& Souza Filho, V. B. (2016). Da guerra às emoções: história da internet e o controverso surgimento do Facebook. Encontro Regional Norte de História da Mídia, n. 4.

Santos e Moura, S. L. dos., Walczak, F. S., \& Moura, R. A. de. (2019). Simulação computacional, essência da agilidade, tecnologia e virtualização com vasta aplicação na indústria manufatureira em geral. In: Congress of Industrial Management and Aeronautical Technology, 1(6), 1-6.

Schlairet, M. C. \& Pollock, J. W. (2010). Teste de equivalência de experiências clínicas tradicionais e simuladas: Aquisição de conhecimento de alunos de graduação em enfermagem. Revista de Educação em Enfermagem, 49(1), 43-47.

Stirling, K., Smith, G., \& Hogg, G. (2012). Os benefícios de um exercício de simulação de enfermaria como uma experiência de aprendizado. British Journal of Nursing, 21(2), 116-122.

Teixeira, I. N. D., \& Felix, J. V C. (2011). Simulación como estrategia de enseñanza de enfermería: revisión de literatura. Interface-comunicação, saúde, educação, 15(39), 1173-1184.

Waxman, K. (2010). O desenvolvimento de cenários de simulação clínica baseados em evidências: Diretrizes para educadores de enfermagem. Jornal da educação em enfermagem, 49(1), 29-35.

Xavier, M. C., Teixeira, C. R., \& Saveti, B. P. (2010). Aplicação das Tecnologias da Informação e Comunicação (TICs) na educação e os desafios do educador. Dialogia, 9(1), 105-116. 